\title{
Evidence Accumulation in Obsessive-Compulsive Disorder: the Role of Uncertainty and Monetary Reward on Perceptual Decision-Making Thresholds
}

\author{
Paula Banca',2,3 , Martin D Vestergaard ${ }^{4}$, Vladan Rankov', Kwangyeol Baek', Simon Mitchell', Tatyana Lapa', \\ Miguel Castelo-Branco ${ }^{3}$ and Valerie Voon ${ }^{*, 1,5,6}$ \\ 'Department of Psychiatry, University of Cambridge, Addenbrooke's Hospital, Cambridge, UK; ${ }^{2}$ PhD Programme in Experimental Biology and \\ Biomedicine, Center for Neuroscience and Cell Biology, University of Coimbra, Portugal; ${ }^{3}$ Institute for Biomedical Imaging and Life Sciences, \\ University of Coimbra, Portugal; ${ }^{4}$ Department of Physiology, Development and Neuroscience, University of Cambridge, Cambridge, UK; \\ ${ }^{5}$ Behavioural and Clinical Neurosciences Institute, University of Cambridge, Cambridge, UK; ${ }^{6}$ Cambridgeshire and Peterborough NHS Foundation \\ Trust, Cambridge, UK
}

\begin{abstract}
The compulsive behaviour underlying obsessive-compulsive disorder (OCD) may be related to abnormalities in decision-making. The inability to commit to ultimate decisions, for example, patients unable to decide whether their hands are sufficiently clean, may reflect failures in accumulating sufficient evidence before a decision. Here we investigate the process of evidence accumulation in $O C D$ in perceptual discrimination, hypothesizing enhanced evidence accumulation relative to healthy volunteers. Twenty-eight $O C D$ patients and thirty-five controls were tested with a low-level visual perceptual task (random-dot-motion task, RDMT) and two response conflict control tasks. Regression analysis across different motion coherence levels and Hierarchical Drift Diffusion Modelling (HDDM) were used to characterize response strategies between groups in the RDMT. Patients required more evidence under high uncertainty perceptual contexts, as indexed by longer response time and higher decision boundaries. HDDM, which defines a decision when accumulated noisy evidence reaches a decision boundary, further showed slower drift rate towards the decision boundary reflecting poorer quality of evidence entering the decision process in patients under low uncertainty. With monetary incentives emphasizing speed and penalty for slower responses, patients decreased the decision thresholds relative to controls, accumulating less evidence in low uncertainty. These findings were unrelated to visual perceptual deficits and response conflict. This study provides evidence for impaired decision-formation processes in OCD, with a differential influence of high and low uncertainty contexts on evidence accumulation (decision threshold) and on the quality of evidence gathered (drift rates). It further emphasizes that OCD patients are sensitive to monetary incentives heightening speed in the speed-accuracy tradeoff, improving evidence accumulation.

Neuropsychopharmacology (20I5) 40, II92-1202; doi:I0.1038/npp.20 I4.303; published online I4 January 2015
\end{abstract}

\section{INTRODUCTION}

The weighting of evidence before a decision can be trivial or can require careful deliberation. The amount of evidence required has much interindividual variability and can be abnormal in psychiatric disorders. The inability to commit to a final decision may be a relevant feature in OCD. In the repetitive act of washing or checking, the available sensoryperceptual evidence appears insufficient to commit to a solid decision: patients appear unable to decide whether their hands are sufficiently clean or the door is properly

*Correspondence: Dr V Voon, Department of Psychiatry, University of Cambridge, Addenbrooke's Hospital, Level E4, Box 189, Hills Road, Cambridge CB2 0QQ, UK, Tel: +44 12237613 27,

E-mail:vv247@cam.ac.uk

Received 18 September 2014; revised 28 October 2014; accepted 7 November 2014; accepted article preview online 26 November 2014 locked (Sachdev and Malhi, 2005). Instead, the compulsive behaviour itself appears to reflect the need for continuous 'evidence gathering', possibly to reduce uncertainty (Rotge et al, 2008; Stern et al, 2013).

The process of accumulating and evaluating evidence before a decision has been assessed using probabilistic reasoning tasks. In the Beads-in-a-Jar task (Beads task) participants judge from which of two jars, containing equal but opposite ratios of colour beads, the beads are being selected (Volans, 1976). In patients with schizophrenia, lower evidence accumulation is consistently observed (Fine et al, 2007; Moutoussis et al, 2011). In the Information Sampling Task participants decide which of two colours is predominant in a $5 \times 5$ matrix by opening boxes to make a decision (Clark et al, 2006). Although both behavioural measures are conceptually similar, recent studies in schizophrenia (Huddy et al, 2013) show impairments in the Beads task but not the Information Sampling Task 
suggesting important task differences. Evidence accumulation in OCD has focused on probabilistic reasoning but the results are mixed. Using the Beads task, two studies (Fear and Healy, 1997; Pelissier and O'Connor, 2002) showed that OCD patients gather more evidence compared with healthy controls, although a third study (Volans, 1976) showed similar findings only after controlling for neuroticism as a confounder. In contrast, a recent study did not replicate this difference (Jacobsen et al, 2012). Using the Information Sampling Task, there were no differences observed in evidence accumulation between OCD patients and controls (Chamberlain et al, 2007).

In this study, we assess evidence gathering in OCD in the perceptual domain. Decisions in daily life are commonly perceptual (for example, do my hands look or feel clean?) rather than probabilistic (for example, are my hands likely to be clean?) or a mix of the two. Typical experimental approaches using sensory discrimination in vibrotactile (Romo and Salinas, 2003), auditory (Kaiser et al, 2007) and visual (for example, using the 'random dot motion' task (RDMT); Newsome et al, 1989) domains have been widely used to investigate perceptual decision-making in primates and healthy humans (for review see (Gold and Shadlen, 2007; Heekeren et al, 2008)). The analysis separates transient sensory integration and decision formation (Gold and Shadlen, 2007). Drift diffusion models, which define a decision when accumulated noisy evidence reaches a criterion level (a decision boundary), have been particularly successful in explaining response time and accuracy data in these binary choice tasks (Ratcliff and McKoon, 2008).

We compared OCD patients and healthy volunteers using the RDMT, a low-level visual perceptual task in which participants decide whether a net of randomly moving dots is predominantly moving right or left. Perceptual and probabilistic decisions are qualitatively different in nature and this is likely to affect patients' cognitive process of evidence accumulation before a decision. One possible difference is that probabilistic tasks (for example, the beads task or Information Sampling Task) require an active accumulation of information (drawing another bead or opening another box), whereas in the perceptual RDMT motor inhibition is necessary (waiting longer results in more evidence accumulation). Motor inhibition as tested using the stop signal task has been shown to be impaired in OCD (Menzies et al, 2007), which may be relevant in any differential impairment of the RDMT. We tested decision thresholds across a range of coherence levels to control for visual processing and compared high and low uncertainty conditions, thus linking decision-making to perceptual uncertainty. The data were analyzed with both conventional regression analysis and computational drift diffusion models. We hypothesized that compared with healthy volunteers, OCD patients would accumulate more perceptual evidence, particularly during high relative to low uncertainty. We also predict that patients with higher compulsive symptoms would exhibit greater impairments in evidence accumulation.

The RDMT has features possibly relevant to response conflict as lower coherence motion may invoke competing responses. In event-related brain potential (ERP) studies, OCD patients have consistently shown enhanced error- related negativity relative to healthy controls (Gehring et al, 2000; Johannes et al, 2001) in response conflict tasks, although variability in behavioural differences has been observed (Marsh et al, 2013; Ursu et al, 2003). This errorprocessing enhancement is localized within the rostral ACC (Fitzgerald et al, 2005; Kiehl et al, 2000) with similar enhanced activity also during correct high conflict (HC) trials, suggesting abnormalities in conflict detection in OCD (Endrass et al, 2008; Ursu et al, 2003). Here we use two different tasks assessing response conflict to act as control tasks: a variation of the Flanker task (Eriksen and Eriksen, 1974), a motor response competition task, modified to enhance task difficulty and a probabilistic selection task (Frank et al, 2007). In this latter task, participants learn three stimulus-pair contingencies during training and are tested on $\mathrm{HC}$ and low conflict (LC) decisions by varying the stimulus reinforcement values using different pairings.

\section{MATERIALS AND METHODS}

\section{Participants}

Sixty-three participants, twenty-eight OCD patients, and thirty-five healthy volunteers (HVs) took part in the study. Recruitment was conducted through community settings and clinicians in East Anglia and advertisements to local support groups. All patients were screened by a psychiatrist, using a structured clinical interview (the Mini International Neuropsychiatric Inventory (Sheehan et al, 1998)), to confirm the OCD diagnosis (DSM-IV-TR criteria; Association AP (2000)) and exclude any comorbid psychiatric disorders. Nineteen of the twenty-eight patients with OCD were taking SSRI medication. Four of them were also taking antipsychotic medication. To assess the severity and characteristics of OCD symptoms, each patient completed the Yale-Brown Obsessive-Compulsive Scale (Y-BOCS) (Goodman et al, 1989). General exclusion criteria for both groups were substance dependence, current major depression of moderate severity, serious neurological, medical or psychiatric illnesses, or head injury. Patients for whom hoarding was the primary complaint were also excluded because hoarding has been recently considered a discrete diagnostic entity due to its significantly different epidemiological, phenomenological, and neurobiological characteristics (Marchand and Phillips McEnany, 2012).

Groups were recruited to match gender, age, and verbal IQ using the National Adult Reading Test (Nelson, 1982). HVs were free from medication or neurological, medical, or psychiatric conditions. All participants completed the Beck Depression Inventory (BDI; Beck et al, 1961) and the State and Trait Anxiety Inventory (Spielberger, 1985).

Participants completed three behavioural tasks in a counterbalanced order and were compensated for their time and performance. The study was approved by the University of Cambridge Research Ethics Committee and written informed consent was obtained.

\section{Behavioural Procedures}

Random-dot motion task (RDMT). Participants viewed a cloud of dots moving within a borderless circle in the screen centre (Figure 1a). The goal was to decide whether the dots 

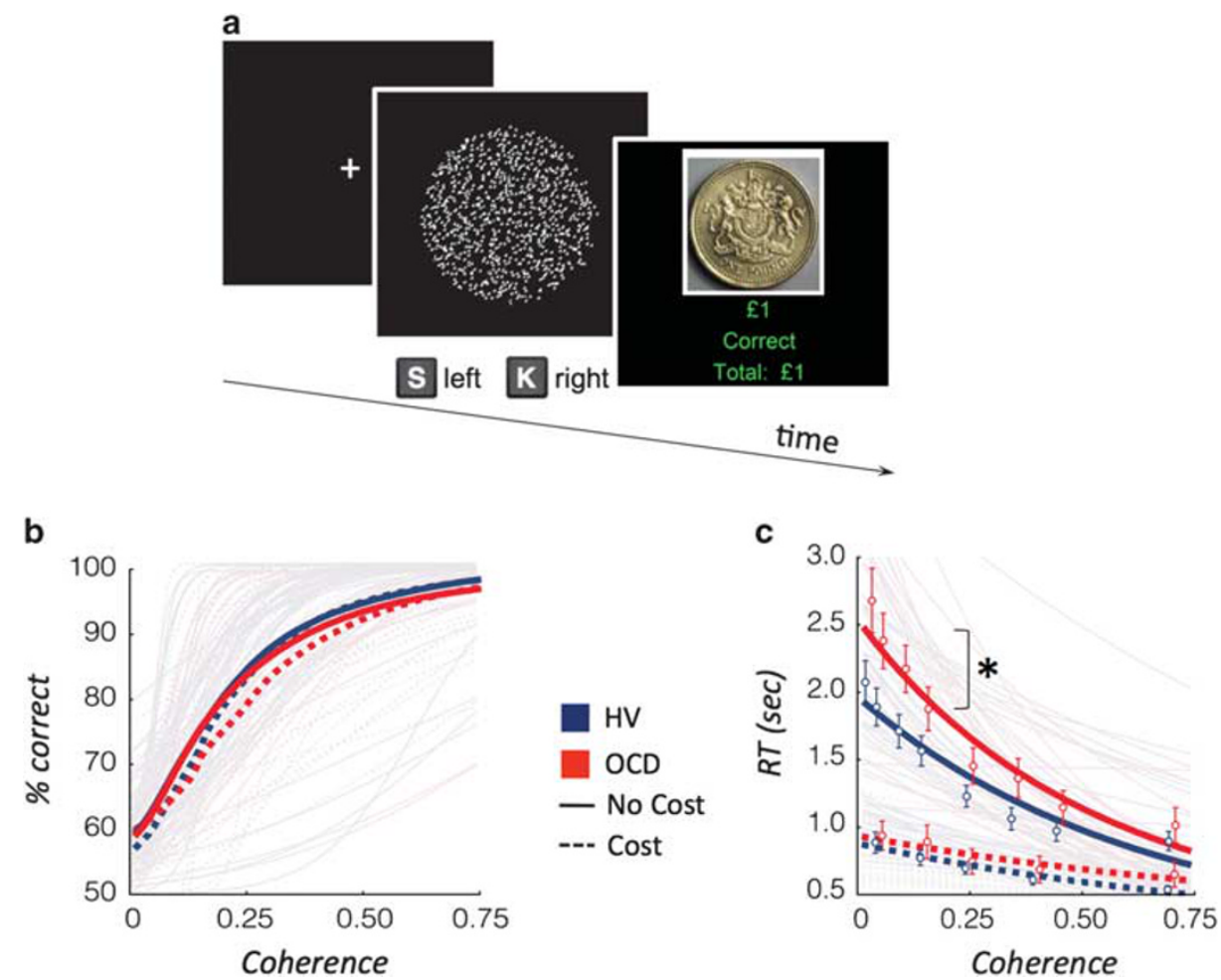

Figure I Random dot motion task and behavioural outcomes. (a) Random-dot motion (RDM) task. Participants viewed a net of dots randomly moving within a borderless circle in the center of the screen. The goal was to decide whether the net of dots appeared to be moving to the right ( $\mathrm{s}$ ) or left ( $\mathrm{k}$ ) direction. (b) Accuracy across the coherence levels for patients with obsessive-compulsive disorder (OCD: red) or healthy volunteers (HV: blue). There were no significant differences when analysed using logistic discrimination of coherent motion. (c) Reaction time (RT) in correctly identified trials across the coherence levels. *RT intercept, $p<0.05$ when analysed using Poisson regression; Open circle: mean average; solid (no cost) and dotted (cost) lines: estimated group averages.

cloud appeared to be moving right or left. Subjects pressed ' $S$ ' for left and ' $K$ ' for right using their index fingers. Two sets of 500 dots (dot size: 3 pixels) were created: the 'coherent set' (dots moving coherently) and the 'random set' (dots moving randomly). From the first 50 -ms frame to the next, the 'coherent set' moved 1 pixel towards the target direction, whereas the 'random set' was randomly reallocated. In the subsequent frame the sets switched, with the 'coherent set' displayed randomly and the 'random set' displayed coherently. This strategy prevented tracking of a specific dot and ensured the cloud remained centred while maintaining the global perception of movement towards one direction (Forstmann et al, 2010). Nine different motion coherence levels were defined by varying the proportion of dots in the 'coherent set'. Coherence levels were selected following extensive piloting to ensure coverage of a wide range of individual visual detection thresholds (coherence levels: 0 (random control condition), $0.025,0.05,0.1,0.15$, $0.25,0.35,0.45$, and 0.7 ) and to ensure representation of high, medium, and low uncertainty. Each trial was followed by an intertrial fixation cross, centred in the middle of the screen, varying between 0.5 and $1 \mathrm{~s}$ duration. The stimulus was displayed for a maximum duration of $10 \mathrm{~s}$ and ceased following a response. Monetary feedback $(+£ 1$ or $-£ 1)$ indicated whether the response was correct or incorrect.

The task consisted of a practice session and three separate conditions. The first condition included nine coherence levels with monetary feedback. The second condition assessed subjective confidence following the decision (six coherence levels). The third condition introduced a monetary penalty (Cost) for slow responses, as well as monetary incentive for fast responses, individualized for reaction time to measure the speed-accuracy tradeoff (six coherence levels). A response time (RT) greater than the individual's average RT +1 SD calculated from the first condition was penalized with $2 £$. Participants received increasing monetary feedback for faster responses $(0.50 £$, $1 £$, or $2 £$ ) and were told that they would receive a proportion of their rewards in the end of the experiment. For further details about each condition, see Supplementary Information.

Primary outcome measures were accuracy, RT, and confidence rates.

\section{Control Tasks}

Two tasks were administered to control for response conflict and are described briefly in the following (see Supplementary Information for a detailed description).

Probabilistic selection task. The Probabilistic selection task has been extensively described involving a training session characterized by choice discrimination of three pairs of stimuli followed by a test phase in which stimuli were paired resulting in either high or LC pairing (Frank et al, 2004) (Figure 4b). 
Flanker task. The Flanker task (Eriksen and Eriksen, 1974; Figure 4a) was modified to enhance task difficulty.

\section{Data Analysis}

Subject characteristics and primary outcomes of the behavioural tasks were analysed using $\chi^{2}$, independent $t$-tests, and mixed-measures ANOVA. The relationship between primary outcome measures and clinical measures was compared using the Pearson correlation.

\section{Random Dot Motion Task}

The RDMT was analysed in detail by using two different approaches focusing on different concepts. A regression analysis accounted for individual visual detection thresholds and assessed performance across all coherence levels. A hierarchical drift diffusion modelling analysis was used to investigate in-depth mechanisms underlying the different response strategies between patients and healthy controls.

Regression analyses. We conducted regression analyses on accuracy and RT assuming coherence level represents the available evidence for detecting motion direction. By only analyzing correctly identified trials, $\log (\mathrm{RT})$ can be interpreted as an index of the cognitive demand required for successful recognition of the motion direction.

Accuracy. Using accuracy as the primary outcome, we used logistic discrimination, $\log \left(\frac{p-0.5}{1-p}\right)=B_{S} C+B_{T}$ to model the identification of coherent motion $(p)$ and characterized behaviour by estimates of visual detection threshold and sensitivity. We used a link function in which odds ratio is characterized in relation to chance performance so that the psychometric function has a lower bound of $50 \%$. Thus, visual detection threshold $\left(-B_{\mathrm{T}} / B_{\mathrm{S}}\right)$ is the coherence level $(C)$ at which $75 \%$ correct identification occurs. $B_{\mathrm{S}}$ measures sensitivity to variation in coherence level. Logistic discrimination assumes binomial distribution of the motion direction identification outcome $(p)$.

Reaction time. Using RT in correctly identified trials as a primary outcome, we then used Poisson regression, $\log (\mathrm{E}(R T \mid C))=B_{C} C+B_{0}$ to model $\mathrm{RT}$ in successful motion direction identification, and characterized behaviour by the intercept and slope. The intercept $\left(B_{0}\right)$ represents the adjusted response for $C=0$ (that is, the expected $\mathrm{RT}$ for zero evidence is $\left.\mathrm{RT}_{0}=\exp \left(B_{0}\right)\right)$. The negative slope parameter $\left(B_{\mathrm{C}}\right)$ indicates the steepness of the exponential decay in RT and represents the release of cognitive demand with increasing coherence level or increasing certainty for motion direction. This regression assumes Poisson distribution of RT. We excluded the random condition $(C=0)$ in the estimation of the parameters.

The generative models were fitted to the behavioural data of each participant using Matlab 2011b Statistics Toolbox $\mathrm{v}$ 7.6. Group differences were tested using $t$-statistics on the parameter estimates across groups.

Hierarchical drift diffusion modelling. We also used Hierarchical Drift Diffusion Model (HDDM) to further explore the mechanisms underlying decisions in the RDMT analysis. This software package (http://ski.clps.brown.edu/ hddm_docs/; Wiecki et al, 2013) allows a fast and flexible estimation of the drift-diffusion model (the most widely used mathematical model of two-alternative forced-choice decision-making tasks (Ratcliff and McKoon, 2008)). In this model each choice is represented as a diffusion towards an upper and lower decision boundary. When the accumulated noisy evidence reaches one of these two boundaries over time, the decision is made and the respective response initiated.

HDDM simultaneously accounts for the proportion of correct and incorrect trials and its respective RT distributions across conditions, considering the latter a result of underlying latent parameters of a decision-making model. It further estimates the posterior probability density of the diffusion model parameters, by using Markov chain Monte Carlo simulation, generating group data, while accounting for individual differences (for details about the model see (Wiecki et al, 2013)). These parameter estimates include: drift rate - the speed of the evidence accumulation process towards either boundary or the quality of the accumulated evidence; decision threshold - the distance between the two boundaries or amount of evidence accumulated; and nondecision time - perceptual encoding and motor execution. The model also allows for a prepotent bias affecting the starting point of the drift process relative to the two boundaries. It then uses analytic integration of the likelihood function for variability in drift-rate and numerical integration for variability in non-decision time and bias.

In this framework, we fit participants' RT and accuracy measures into the model. We compared drift rate and decision threshold. We used all coherence levels in the analysis comparing high (0.025 and 0.05), medium (0.15 and 0.25$)$, and low (0.45 and 0.7$)$ levels of uncertainty. We compared the proportion $(q)$ of posterior distributions that overlap between groups in the high, medium, and low uncertainty conditions and assess if less than $5 \%$ of the distribution overlaps (Wiecki et al, 2013). For the main hypothesis, to account for multiple comparisons, we assigned significance if less than $0.8 \%$ (or $q<0.008$ ) of the distributions overlapped (Bonferroni correction).

We tested the hypothesis that OCD patients would show a more cautious style of responding represented by a higher decision threshold and/or a slower drift rate.

\section{Control Tasks}

Probabilistic selection task. For the training phase, we assessed both accuracy and learning consistency. Consistency was defined for individual performance in terms of how well the actual performance reflected the performance of an optimal learner taking into account the actual evidence observed. The accuracy score is based on the prior probability of the options, whereas the consistency score is based on the posterior probability of the options. Thus, the consistency score is a more accurate score of performance as an index of learning. We also assessed measures of win-stay or lose-switch. Group differences were analysed using independent $t$-tests.

For the testing phase we used a mixed measures ANOVA with Group as a between-subjects factor and Conflict as a within-subjects factor, to assess accuracy and RT on correct trials. 
Flanker task. In the Flanker task, the mean RT for correct trials and accuracy were computed for each visual context (congruent and incongruent) and each stimulus type (arrow and letters). These measures were analysed using an ANOVA with the following factors: group, visual context, and stimulus type, in which the last two were specified as repeated measures.

\section{RESULTS}

Twenty-eight OCD subjects and thirty-five HVs were assessed. Table 1 summarizes the groups' demographic and clinical characteristics.

Table I Demographic and Clinical Characteristics of the Participants

\begin{tabular}{|c|c|c|c|c|c|}
\hline & \multirow{2}{*}{$\begin{array}{c}\text { OCD } \\
(n=28)\end{array}$} & \multirow{2}{*}{$\begin{array}{c}\text { HV } \\
(n=35)\end{array}$} & \multicolumn{3}{|c|}{ Statistics } \\
\hline & & & $\mathbf{t}$ & df & $p$ \\
\hline Gender (female/male) & $16 / 12$ & $20 / 15$ & & & \\
\hline Age & $37.5(13.5)$ & $37.9(14.7)$ & 0.141 & 61 & ns \\
\hline Verbal IQ & | I $5.4(5.8)$ & | $18.4(6.0)$ & 1.705 & 45 & ns \\
\hline Y-BOCS total & $24.3(6.9)$ & - & & & \\
\hline Obsessions & $12.6(3.8)$ & - & & & \\
\hline Compulsions & 12.1 (3.2) & - & & & \\
\hline OCS & - & $9.88(3.31)$ & & & \\
\hline $\mathrm{OCl}-\mathrm{R}$ & - & $9.76(8.91)$ & & & \\
\hline $\mathrm{BDI}$ & $18.0(10.1)$ & $4.4(4.7)$ & -6.498 & 60 & $<0.001$ \\
\hline STAI-S & $46.9(12.5)$ & $32.7(10.4)$ & -4.826 & 59 & $<0.001$ \\
\hline STAI-T & $54.4(11.7)$ & $35.2(10.8)$ & -6.581 & 58 & $<0.001$ \\
\hline
\end{tabular}

Abbreviations: BDI, Beck Depression Inventory; HV, healthy volunteers; NART, National Adult Reading Test; OCD, patient group; STAI-S, State component of State-Trait Anxiety Inventory; STAI-T, Trait component of State-Trait Anxiety Inventory; Y-BOCS, Yale-Brown Obsessive Compulsive Scale (total, obsession, and compulsion scores).

Standard deviations are in parentheses: mean (SD).

The different degrees of freedom ( $d f$ resulted from missing data in the data set.

\section{Random-Dot Motion Task}

The data from two OCD participants were excluded as their performance was at chance level throughout the task.

\section{Regression Analyses}

Logistic discrimination analysis showed no differences in the visual detection threshold and sensitivity parameters between groups (Figure $1 \mathrm{~b}$ ), meaning that accuracy and the sensitivity to variation in the coherence level were similar across groups. However, as predicted in our primary hypothesis, the Poisson regression analysis showed that $\mathrm{RT}$ intercept, but not the slope, differed between groups $(t=2.37, \mathrm{df}=59, p=0.021$; Figure $1 c$; for secondary analysis please see Table 2). As the RT intercept occurs at low coherence level, this result is consistent with OCD patients being slower than HV under higher uncertainty.

The regression analysis in the Cost condition showed no group differences (Table 2).

There was no correlation between visual detection threshold and RT intercept (HV: $p=0.68$; OCD: $p=0.33$ ), suggesting that visual detection threshold was unrelated to these findings.

A positive correlation was found between the Y-BOCS compulsive subscale scores and the visual detection threshold $(r=0.48, p=0.015)$ and the RT slope $(r=0.40$, $p=0.047$; exploratory analysis between RDMT parameters and Y-BOCS subscales are shown in Table 3). Thus, the

Table 3 Correlations Between the Parameters Estimated with Regression Analysis of the Random-dot Motion Task (no cost condition) and Y-BOCS Measure

\begin{tabular}{lcr}
\hline \multicolumn{3}{c}{ Correlations: $\boldsymbol{r}(\boldsymbol{p}$-value $)$} \\
\hline Parameters & Y-COMP & \multicolumn{1}{c}{ Y-OBS } \\
\hline Vis. detection threshold $\left(-\boldsymbol{B}_{\mathbf{T}} / \mathbf{B}_{\mathbf{S}}\right)$ & $0.48(0.015)$ & $0.38(0.06)$ \\
Sensitivity $\left(\boldsymbol{B}_{\mathbf{S}}\right)$ & $-0.01(0.95)$ & $-0.15(0.47)$ \\
RT intercept $\left(\boldsymbol{B}_{\mathbf{0}}\right)$ & $-0.25(0.22)$ & $-0.02(0.89)$ \\
Slope $\left(\boldsymbol{B}_{\mathbf{C}}\right)$ & $0.40(0.047)$ & $0.18(0.38)$ \\
\hline
\end{tabular}

Abbreviations: YOBS, Y-BOCS obsessive subscale; YCOMP, Y-BOCS compulsive subscale.

Table 2 Random-dot Motion Task Statistics for the Regression Analysis

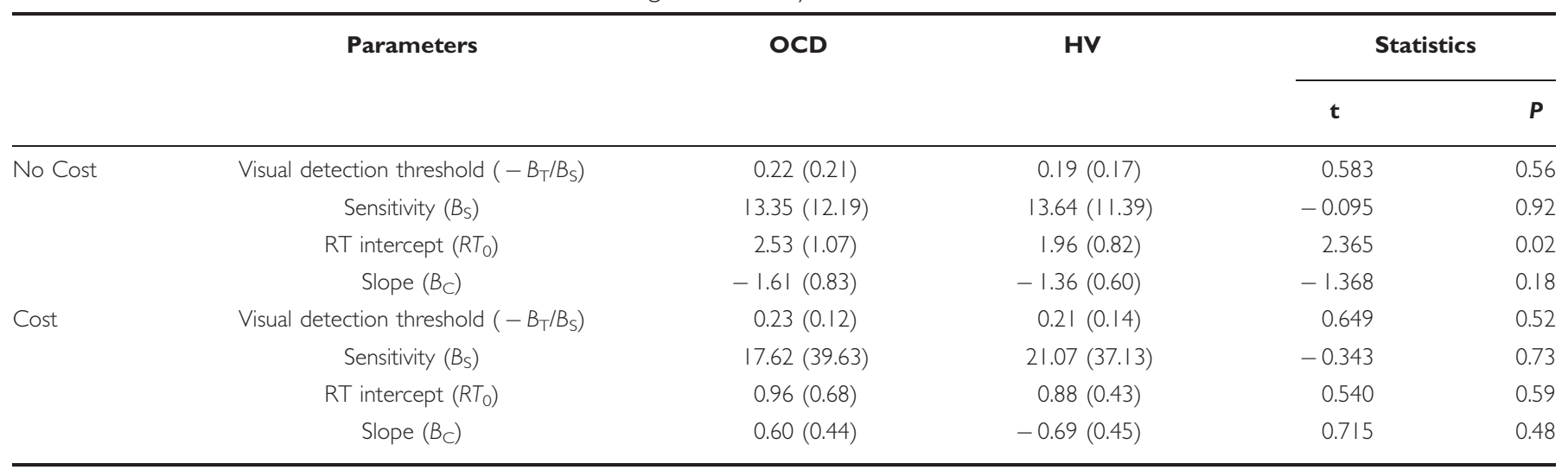

Abbreviations: $\mathrm{HV}$, healthy volunteers; OCD, patient group.

Standard deviations are in parentheses: mean (SD). 
more severe compulsive symptoms, the higher the motion detection thresholds and the steepness of the exponential decay in $R T$. There were no other significant correlations between the RDMT parameters and clinical measures or the outcome measures from other tasks.

We assessed baseline reaction time using the successful RT from coherence level $=0.7$ to study the possible effect of neuroleptic medication. As no significant results were found $(p=0.88)$ between patients on and off neuroleptic medication, we assume that this medication did not interfere in the RT.

\section{Hierarchical Drift Diffusion Modelling}

HDDM analysis showed that OCD patients had higher decision thresholds than $\mathrm{HV}$ in conditions under higher uncertainty (proportion of overlap of posterior distributions: high uncertainty: $q<0.0001$; medium uncertainty: $q<0.0001$; low uncertainty: $q=0.16$; Figure 2). However, the drift rate was slower in OCD patients as compared with $\mathrm{HV}$ only in low and medium uncertainty $(q=0.001)$ but not in high uncertainty $(q=0.05$; Bonferroni corrected $q<0.008)$.

There was an effect of cost on decision threshold and drift rate. In the Cost condition, OCD subjects had similar thresholds to $\mathrm{HV}$ in high uncertainty $(q=0.83)$ and medium uncertainty $(q=0.86)$ and lower thresholds in low uncertainty $(q<0.001$; Figure 3$)$. With cost, OCD patients continued to show slower drift rates compared with $\mathrm{HV}$ in low uncertainty $(q<0.0001)$ but not in medium and high uncertainty $(q=0.21)$.

\section{Confidence and Post-Feedback RT}

There were no significant differences in confidence or postfeedback RT (see Supplementary Information and Supplementary Figure S1).

\section{Control Tasks}

Probabilistic selection task. In the training phase, there were no differences between groups in the consistency score, RT, or win-stay or lose-switch measures. In the testing phase, there were no Group effects or Group $\times$ Conflict effects on accuracy or RT (see Supplementary Information). Results are shown in Figure $4 \mathrm{~b}$.

Flanker task. Three participants were not tested on this task due to time constraints. Thus, the following results are from 26 OCD participants and $34 \mathrm{HV}$. There were no Group or Group $\times$ Visual context or Group $\times$ Stimulus type interaction effects on accuracy or RT (see Supplementary Information). Results are shown in Figure 4a.

Relationship between behavioural tasks. There were no correlations between the parameter estimates of the three behavioural tasks.

\section{DISCUSSION}

Using a visual perceptual task, we found that compared with HVs, OCD patients were more cautious in weighing the alternatives before making a decision, particularly when decisions involved higher perceptual uncertainty. Higher compulsivity scores were associated with greater impairments in evidence accumulation with higher motion detection thresholds and slower performance. In the HDDM analysis, OCD subjects had higher decision boundaries (that is, they needed more evidence to make a decision), particularly under high uncertainty. However, the HDDM analysis also showed slower drift rate in OCD patients particularly under low uncertainty. The drift rate is a measure of the speed of accumulation of evidence over time and represents the strength or quality of evidence from the stimulus entering the decision process (Ratcliff and
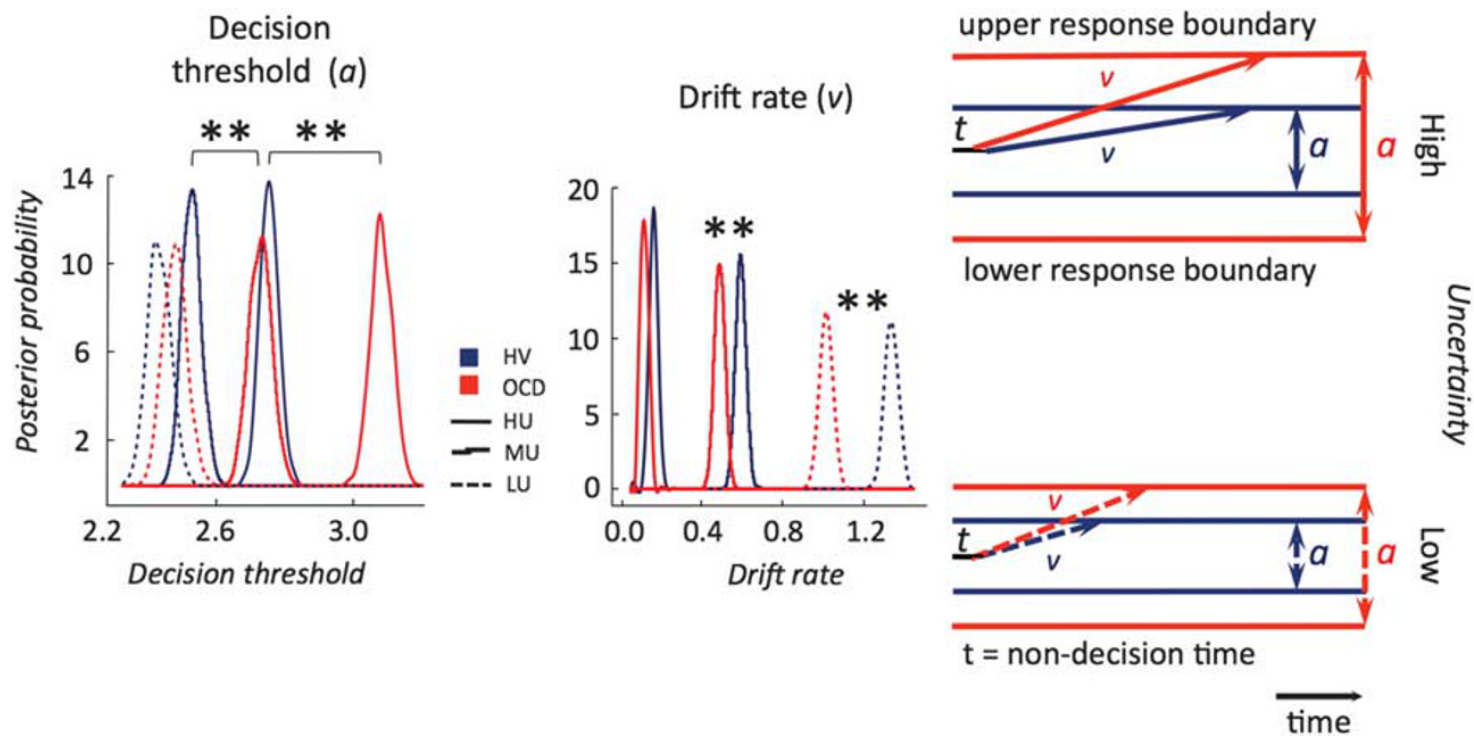

Figure 2 Hierarchical drift diffusion modelling of random dot motion task: No Cost condition. Posterior density plots of the group means of the decision thresholds and drift-rates for No Cost condition and their schematic representation (on the right). Red lines: Obsessive-compulsive disorder (OCD); blue lines: healthy volunteers $(\mathrm{HV})$; solid lines: high uncertainty $(\mathrm{HU})$; Stepped lines: medium uncertainty (MU); Dashed lines: low uncertainty (LU); **overlap of posterior distribution $<0.001$. 
Decision threshold (a)
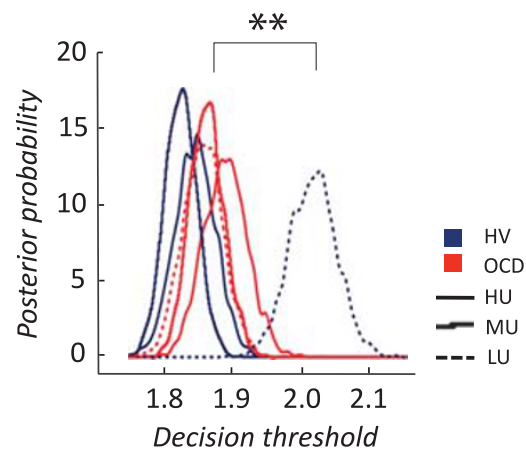

Drift rate $(v)$

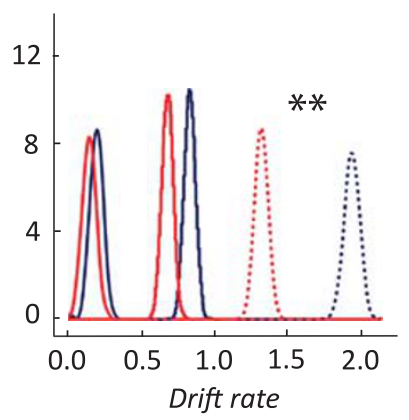

upper response boundary

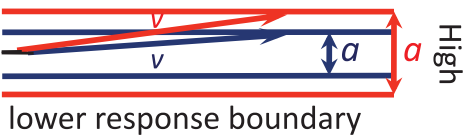

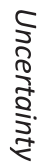

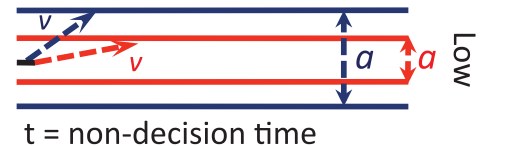

$\overrightarrow{\text { time }}$

Figure 3 Hierarchical drift diffusion modelling of random dot motion task: Cost condition. Posterior density plots of the group means of the decision thresholds and drift-rates for Cost condition and their schematic representation (on the right). Red lines: obsessive-compulsive disorder (OCD); blue lines: healthy volunteers $(\mathrm{HV})$; solid lines: high uncertainty $(\mathrm{HU})$; stepped lines: medium uncertainty (MU); dashed lines: low uncertainty (LU); **overlap of posterior distribution $<0.001$.

a

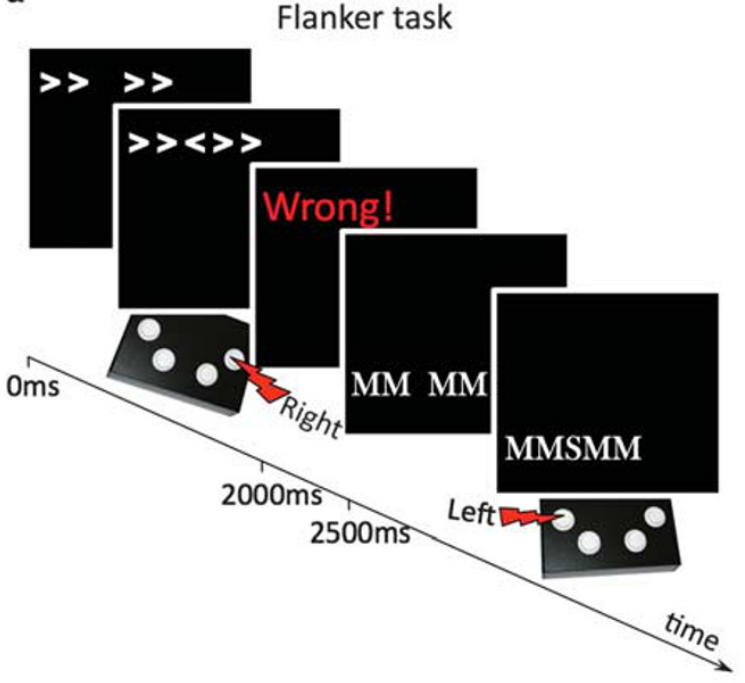

b

Probabilistic selection task
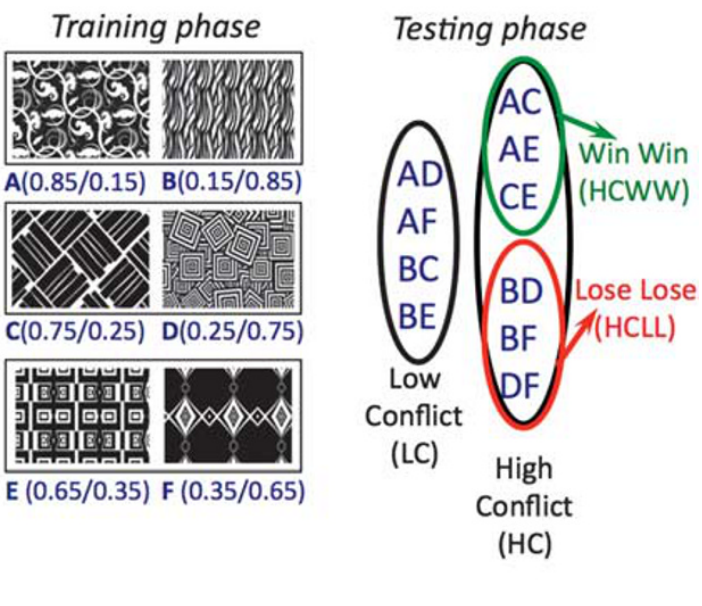

(HC)

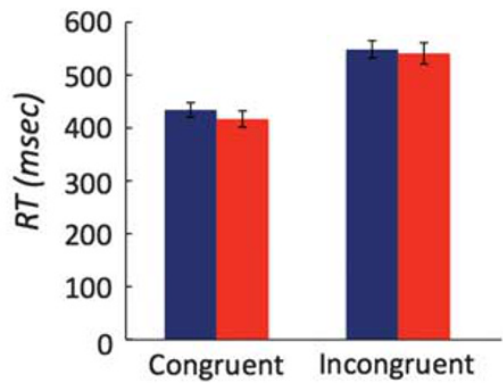

HV

OCD

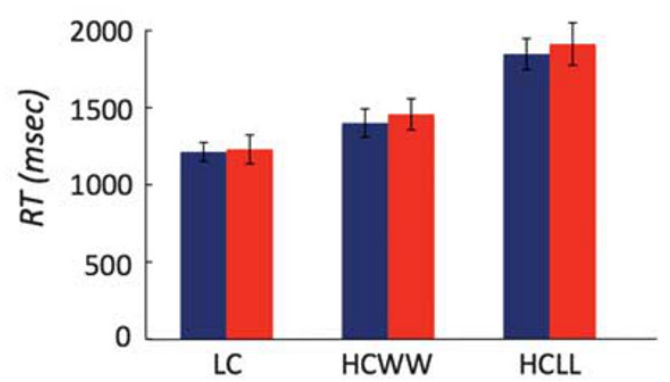

Figure 4 Flanker task and probabilistic selection task. (a) Flanker task. Top: subjects viewed a string of arrows or letters varying in screen position. The goal was to indicate the direction (left: arrow or S; right: arrow or $\mathrm{M}$ ) of the central character flanked by either the same (congruent) or different (incongruent) flankers using the left or right button. Bottom: mean response time (RT) for congruent and incongruent conditions for Obsessive compulsive disorder (OCD) and healthy volunteers (HVs). (b) Probabilistic selection task. Top: During training, subjects learned stimulus contingencies from randomly presented stimulus pairs from three probability configurations followed by monetary feedback for correct and incorrect choices. Correct choices were determined probabilistically (ratio of positive/negative monetary feedback is shown in parentheses for each stimulus). In the testing phase novel combinations were presented to assess high and low conflict decisions. This schematic illustration was adapted from Frank et al, (2007). Bottom: mean RT for low conflict (LC), high conflict win-win (HCWW), and high conflict lose-lose (HCLL) conditions in OCD and HV. Error bars represent SEM. 
McKoon, 2008). These findings emphasize the compatibility and added value of the behavioural and computational model analyses. Our findings further highlight that by emphasizing speed (in the Cost condition of the RDMT), OCD subjects normalized their decision reaction time and reversed the difference in decision boundaries, thus improving evidence accumulation and speed and possibly shifting away from a pathological internal monitoring without compromising accuracy (as shown with the logistic regression analysis).

\section{Random Dots Motion Task}

Several features make this low-level perceptual task an optimal paradigm to study evidence accumulation in OCD. First, it has been extensively investigated in primate studies and healthy humans (Gold and Shadlen, 2007; Heekeren et al, 2008). Second, by using neutral and nonthreatening stimuli, decision accumulation is assessed without confounding OCD-relevant stimuli. Third, in studies manipulating dot motion-viewing time, decision accuracy improved as a function of motion-viewing duration (Gold and Shadlen, 2007), contrary to other perceptual tasks in which the two were unrelated (Uchida et al, 2006). Thus, RDMT reaction time is representative of decision-making. Fourth, patients with OCD have reported difficulties with high-level perceptual tasks (Savage et al, 1999; Kim et al, 2008; Shin et al, 2013) but no differences in low-level visual processing (RDMT) have been observed (Kim et al, 2008). We further confirm that visual detection thresholds were similar between groups. Finally, this task controls for working memory by using a single stimulus presentation. Other perceptual paradigms assessed in OCD have studied working memory (Lambrecq et al, 2013), perceptual visual deficits (Kim et al, 2008), and uncertainty (Rotge et al, 2008; Toffolo et al, 2013; van den Hout et al, 2009) without specifically focusing on the degree of uncertainty or evaluating decision formation. Many of these tasks did not control for working memory, which may be particularly relevant in OCD given reported impairments in working memory (Chamberlain et al, 2007; Morein-Zamir et al, 2010) and in memory confidence and distrust (Dar, 2004; Hermans et al, 2008; van den Hout et al, 2009). Thus, the RDMT measures cognitive evidence accumulation without the relevant confounders of working memory or high-level visual perception.

Probabilistic reasoning studies in OCD have previously shown inconsistent results (Fear and Healy, 1997; Jacobsen et al, 2012). Perceptual decision tasks may be more ecologically valid as many daily decisions are based on sensory ambiguity rather than probabilistic evaluations. Perceptual tasks have been applied to HVs with high OC scores (a visual search task focusing on absent targets with presumably greater uncertainty (Toffolo et al, 2013) and a perceptual colour judgement task), showing prolonged RT and increased indecisiveness along with greater feedback requests (Sarig et al, 2012). Here we focused specifically on OCD patients. Our study also controlled for possible related explanations related to conflict monitoring, by considering both perceptual and probabilistic types of conflict. We did not find any group differences in the Flanker task or probabilistic selection task or any correlations between conflict outcome measures and the RDMT.

\section{Speed-Accuracy Tradeoff}

Here we show that by emphasizing speed over accuracy we eliminate and indeed reverse differences in evidence accumulation with no differences in accuracy between groups. The drift rate hastens and decision boundaries decrease across all subjects. However, although OCD subjects still have slower drift rates under low uncertainty conditions, they markedly improve the evidence accumulation with lower decision boundaries compared with HVs. In other words, although patients were still impaired in their implicit experience of the quality of evidence and were slower to reach the decision boundary, they required less evidence to make a decision and did not sacrifice accuracy.

Our results contrast with a study with the Information Sampling Task showing that OCD patients were not sensitive to point penalty for evidence accumulation (Chamberlain et al, 2007), which may be less salient than monetary penalty. Our results suggest that in OCD subjects explicit salient incentives on evidence accumulation (speed or information sampling) may be implicitly incorporated into and shift the internal cost-benefit signals during the evidence accumulation process, presumably shifting away from pathological internal monitoring. That we did not find an influence on outcome parameters following positive or negative feedback further emphasizes a differential role of implicit cost and external feedback in decision formation in OCD, possibly consistent with a reliance in OCD patients on internal signals rather than external feedback.

\section{The Role of Uncertainty}

We demonstrated a differential influence of high and low objective uncertainty contexts on evidence accumulation and on the quality of evidence. Both high and low uncertainty may be relevant in OCD; high uncertainty may reflect situations such as exposure to biological fluids from an ill individual and low uncertainty the compulsive behaviour even after repeated hand washing. Both the regression analysis and decision boundary differences (amount of evidence accumulated) in the HDDM analysis emphasized an effect of high uncertainty, whereas the HDDM analysis further emphasized differences in drift rate as a function of low uncertainty. The drift rate or speed of evidence accumulation provides a qualitatively different measure representing the strength or quality of evidence from the stimulus entering the decision process. Other studies have also shown impairments in OCD as a function of uncertainty; using a delayed matching-to-sample task with unrestricted choice verification, poor insight triggered greater checking behaviours in OCD patients, which indexed uncertainty (Jaafari et al, 2011; Rotge et al, 2008).

OCD subjects have also shown greater explicit subjective ratings of uncertainty for low but not higher uncertainty evidence in a probabilistic reasoning task (Stern et al, 2013). The subjective levels of certainty assessed intermediate levels of evidence, whereas we assessed subjective decision confidence levels and did not show any differences. Some (Dar, 2004; Stern et al, 2012) but not all studies (Sarig et al, 2012) have shown impairments in subjective certainty in OCD. Patients have also been shown to be more risk averse under low risk (defined as variance with a lower difference 
between positive and negative OCD-relevant outcomes) as compared with high-risk situations. Although our current study has broad similarities on the role of low uncertainty, we focused on neutral stimuli under uncertainty (in which probabilities are unknown) as compared with OCD-relevant outcomes of risk (in which the probabilities are known).

Although OCD checking may be motivated by the wish to reduce uncertainty, checking compulsions appear to have the opposite effect, fostering doubt, greater uncertainty and meta-memory problems (Hermans et al, 2008; van den Hout and Kindt, 2003a,b). This paradoxical effect might be a consequence of deficits in memory confidence, which ironically appears to result from the checking behaviour itself (Rotge et al, 2012; van den Hout et al, 2009). OC-like perseveration itself has been suggested to impair memory and perception distrust. For instance, prolonged visual attention to stimuli provokes feelings of dissociation and uncertainty about perception (van den Hout et al, 2008). Thus, shifting the attentional focus may secondarily improve perceptual uncertainty and may have therapeutic relevance.

\section{Behavioural and Computational Analyses}

The behavioural and HDDM analyses were highly consistent. The regression analysis commonly used in visual processing studies, which conventionally focuses on successful trials, highlighted a role for high uncertainty. This analysis accounts for visual detection thresholds and fit across all coherence levels for successful trials. Similarly, HDDM, which models both successful and unsuccessful trials, showed greater decision boundaries, which was particularly enhanced under high uncertainty. HDDM has the potential to characterize the mechanism underlying these results by further estimating the latent parameters, drift rate, and decision boundaries based on the distributions of RT for both correct and incorrect trials (Wiecki et al, 2013). A specific effect of low uncertainty on drift rate was shown in the HDDM analysis, which may be in part related to the inclusion of incorrect trials in the HDDM analysis. In OCD subjects, incorrect trials as assessed in conflict tasks using ERP measures are associated with a robust enhancement in error-related negativity, whereas correct trials are associated with a weaker increase in correct related negativity (Endrass et al, 2008). This enhanced monitoring of incorrect responses may also be reflected in the implicit assessment of the evidence quality, which may be more relevant in ambiguous or low uncertainty situations. Thus, by including all possible outcomes in the HDDM analysis, a more comprehensive picture may emerge relative to the behavioural analysis.

\section{CONCLUSION}

Compulsive behaviours in OCD may be an attempt to accumulate sufficient evidence to commit to a decision and may be influenced by the degree of uncertainty. Deep brain stimulation targeting the subthalamic nucleus has shown an influence on the RDMT in patients with Parkinson's disease (Green et al, 2013) but not on a perceptual decision task (Djamshidian et al, 2014); further studies exploring these differences and particularly in OCD patients may be indicated. Our results highlight the differential role of implicit incentives and external feedback in decision formation in OCD. We show that OCD subjects are sensitive to monetary incentives emphasizing speed in evidence accumulation, shifting the speed-accuracy tradeoff away from the pathological internal monitoring without sacrificing accuracy. This capacity to shift may enable new therapeutic approaches.

\section{FUNDING AND DISCLOSURE}

The material is original research, has not been previously published and has not been submitted for publication elsewhere. PB was supported by the Portuguese Foundation for Science and Technology (individual fellowship to PB: SFRH/BD/33889/2009). VV is a Wellcome Trust Intermediate Fellow in Clinical Neurosciences (093705/Z/10/Z). MDV was supported by the Wellcome Trust. The remaining authors declare no competing financial interests. The authors declare no conflict of interest.

\section{ACKNOWLEDGEMENTS}

We would like to acknowledge all the participants for their participation in this study. We would also like to thank Michael J Frank for helpful discussions on the HDDM data.

\section{REFERENCES}

Association AP (2000). Diagnostic and statistical manual of mental disorders: DSM-IV-TR. American Psychiatric Association: Washington, DC.

Beck AT, Ward CH, Mendelson M, Mock J, Erbaugh J (1961). An inventory for measuring depression. Arch Gen Psychiatry 4: 561-571.

Chamberlain SR, Fineberg NA, Blackwell AD, Clark L, Robbins TW, Sahakian BJ (2007). A neuropsychological comparison of obsessive-compulsive disorder and trichotillomania. Neuropsychologia 45: 654-662.

Clark L, Robbins TW, Ersche KD, Sahakian BJ (2006). Reflection impulsivity in current and former substance users. Biol Psychiatry 60: 515-522.

Dar R (2004). Elucidating the mechanism of uncertainty and doubt in obsessive-compulsive checkers. J Behav Ther Exp Psychiatry 35: 153-163.

Djamshidian A, O’Sullivan SS, Tomassini A, Foltynie T, Limousin P, Aviles-Olmos I et al (2014). In a rush to decide: deep brain stimulation and dopamine agonist therapy in Parkinson's disease. J Parkinsons Dis 4: 579-583.

Endrass T, Klawohn J, Schuster F, Kathmann N (2008). Overactive performance monitoring in obsessive-compulsive disorder: ERP evidence from correct and erroneous reactions. Neuropsychologia 46: 1877-1887.

Eriksen BA, Eriksen CW (1974). Effects of noise letters upon identification of a target letter in a non-search task. Percept Psychophys 16: 143-149.

Fear CF, Healy D (1997). Probabilistic reasoning in obsessivecompulsive and delusional disorders. Psychol Med 27: 199-208.

Fine C, Gardner M, Craigie J, Gold I (2007). Hopping, skipping or jumping to conclusions? Clarifying the role of the JTC bias in delusions. Cogn Neuropsychiatry 12: 46-77.

Fitzgerald KD, Welsh RC, Gehring WJ, Abelson JL, Himle JA, Liberzon I et al (2005). Error-related hyperactivity of the 
anterior cingulate cortex in obsessive-compulsive disorder. Biol Psychiatry 57: 287-294.

Forstmann BU, Anwander A, Schafer A, Neumann J, Brown S, Wagenmakers EJ et al (2010). Cortico-striatal connections predict control over speed and accuracy in perceptual decision making. Proc Natl Acad Sci USA 107: 15916-15920.

Frank MJ, Samanta J, Moustafa AA, Sherman SJ (2007). Hold your horses: impulsivity, deep brain stimulation, and medication in parkinsonism. Science 318: 1309-1312.

Frank MJ, Seeberger LC, O’Reilly RC (2004). By carrot or by stick: cognitive reinforcement learning in parkinsonism. Science 306: 1940-1943.

Gehring WJ, Himle J, Nisenson LG (2000). Action-monitoring dysfunction in obsessive-compulsive disorder. Psychol Sci 11: $1-6$.

Gold JI, Shadlen MN (2007). The neural basis of decision making. Annu Rev Neurosci 30: 535-574.

Goodman WK, Price LH, Rasmussen SA, Mazure C, Fleischmann RL, Hill CL et al (1989). The Yale-Brown Obsessive Compulsive Scale. I. Development, use, and reliability. Arch Gen Psychiatry 46: $1006-1011$.

Green N, Bogacz R, Huebl J, Beyer AK, Kuhn AA, Heekeren HR (2013). Reduction of influence of task difficulty on perceptual decision making by STN deep brain stimulation. Curr Biol 23: 1681-1684.

Heekeren HR, Marrett S, Ungerleider LG (2008). The neural systems that mediate human perceptual decision making. Nat Rev Neurosci 9: 467-479.

Hermans D, Engelen U, Grouwels L, Joos E, Lemmens J, Pieters G (2008). Cognitive confidence in obsessive-compulsive disorder: distrusting perception, attention and memory. Behav Res Ther 46: $98-113$.

Huddy VC, Clark L, Harrison I, Ron MA, Moutoussis M, Barnes TR et al (2013). Reflection impulsivity and response inhibition in first-episode psychosis: relationship to cannabis use. Psychol Med 43: 2097-2107.

Jaafari N, Aouizerate B, Tignol J, El-Hage W, Wassouf I, Guehl D et al (2011). The relationship between insight and uncertainty in obsessive-compulsive disorder. Psychopathology 44: 272-276.

Jacobsen P, Freeman D, Salkovskis P (2012). Reasoning bias and belief conviction in obsessive-compulsive disorder and delusions: jumping to conclusions across disorders? $\mathrm{Br} \mathrm{J}$ Clin Psychol 51: 84-99.

Johannes S, Wieringa BM, Nager W, Rada D, Dengler R, Emrich HM et al (2001). Discrepant target detection and action monitoring in obsessive-compulsive disorder. Psychiatry Res 108: $101-110$.

Kaiser J, Lennert T, Lutzenberger W (2007). Dynamics of oscillatory activity during auditory decision making. Cereb Cortex 17: 2258-2267.

Kiehl KA, Liddle PF, Hopfinger JB (2000). Error processing and the rostral anterior cingulate: an event-related fMRI study. Psychophysiology 37: 216-223.

Kim J, Blake R, Park S, Shin YW, Kang DH, Kwon JS (2008). Selective impairment in visual perception of biological motion in obsessive-compulsive disorder. Depress Anxiety 25: E15-E25.

Lambrecq V, Rotge JY, Jaafari N, Aouizerate B, Langbour N, Bioulac B et al (2013). Differential role of visuospatial working memory in the propensity toward uncertainty in patients with obsessive-compulsive disorder and in healthy subjects. Psychol Med 1-12.

Marchand S, Phillips McEnany G (2012). Hoarding's place in the DSM-5: another symptom, or a newly listed disorder? Issues Ment Health Nurs 33: 591-597.

Marsh R, Horga G, Parashar N, Wang Z, Peterson BS, Simpson HB (2013). Altered activation in fronto-striatal circuits during sequential processing of conflict in unmedicated adults with obsessive-compulsive disorder. Biol Psychiatry 75: 615-625.
Menzies L, Achard S, Chamberlain SR, Fineberg N, Chen $\mathrm{CH}$, del Campo $\mathrm{N}$ et al (2007). Neurocognitive endophenotypes of obsessive-compulsive disorder. Brain 130(Pt 12): 3223-3236.

Morein-Zamir S, Craig KJ, Ersche KD, Abbott S, Muller U, Fineberg NA et al (2010). Impaired visuospatial associative memory and attention in obsessive compulsive disorder but no evidence for differential dopaminergic modulation. Psychopharmacology 212: 357-367.

Moutoussis M, Bentall RP, El-Deredy W, Dayan P (2011). Bayesian modelling of Jumping-to-Conclusions bias in delusional patients. Cogn Neuropsychiatry 16: 422-447.

Nelson HE (1982). National Adult Reading Test (NART): Test Manual NFER-Nelson: Windsor. UK.

Newsome WT, Britten KH, Movshon JA (1989). Neuronal correlates of a perceptual decision. Nature 341: 52-54.

Pelissier MC, O'Connor KP (2002). Deductive and inductive reasoning in obsessive-compulsive disorder. $\mathrm{Br} \mathrm{J} \mathrm{Clin} \mathrm{Psychol}$ 41(Pt 1): 15-27.

Ratcliff R, McKoon G (2008). The diffusion decision model: theory and data for two-choice decision tasks. Neural Comput 20: 873-922.

Romo R, Salinas E (2003). Flutter discrimination: neural codes, perception, memory and decision making. Nat Rev Neurosci 4: 203-218.

Rotge JY, Clair AH, Jaafari N, Hantouche EG, Pelissolo A, Goillandeau $\mathrm{M}$ et al (2008). A challenging task for assessment of checking behaviors in obsessive-compulsive disorder. Acta Psychiatr Scand 117: 465-473.

Rotge JY, Langbour N, Dilharreguy B, Bordessoulles M, Guehl D, Bioulac B et al (2012). Contextual and behavioral influences on uncertainty in obsessive-compulsive disorder. Cortex 23: 00346-2.

Sachdev PS, Malhi GS (2005). Obsessive-compulsive behaviour: a disorder of decision-making. Aust N Z J Psychiatry 39: 757-763.

Sarig S, Dar R, Liberman N (2012). Obsessive-compulsive tendencies are related to indecisiveness and reliance on feedback in a neutral color judgment task. J Behav Ther Exp Psychiatry 43: 692-697.

Savage CR, Baer L, Keuthen NJ, Brown HD, Rauch SL, Jenike MA (1999). Organizational strategies mediate nonverbal memory impairment in obsessive-compulsive disorder. Biol Psychiatry 45: 905-916.

Sheehan DV, Lecrubier Y, Sheehan KH, Amorim P, Janavs J, Weiller E et al (1998). The Mini-International Neuropsychiatric Interview (M.I.N.I.): the development and validation of a structured diagnostic psychiatric interview for DSM-IV and ICD-10. J Clin Psychiatry 59(Suppl 20): 22-33.

Shin NY, Jang JH, Kim HS, Shim G, Hwang JY, Kim SN et al (2013). Impaired body but not face perception in patients with obsessive-compulsive disorder. J Neuropsychol 7: 58-71.

Spielberger CD (1985). Assessment of state and trait anxiety: Conceptual and methodological issues. South Psychologist 2: 6-16.

Stern ER, Welsh RC, Gonzalez R, Fitzgerald KD, Abelson JL, Taylor SF (2012). Subjective uncertainty and limbic hyperactivation in obsessive-compulsive disorder. Hum Brain Mapp 34: 1956-1970.

Stern ER, Welsh RC, Gonzalez R, Fitzgerald KD, Abelson JL, Taylor SF (2013). Subjective uncertainty and limbic hyperactivation in obsessive-compulsive disorder. Hum Brain Mapp 34: 1956-1970.

Toffolo MBJ, Van den Hout MA, Hooge ITC, Engelhard IM, Cath DC (2013). Mild uncertainty promotes checking behavior in subclinical obsessive-compulsive disorder. Clin Psychol Sci 1: 103-109.

Uchida N, Kepecs A, Mainen ZF (2006). Seeing at a glance, smelling in a whiff: rapid forms of perceptual decision making. Nat Rev Neurosci 7: 485-491.

Ursu S, Stenger VA, Shear MK, Jones MR, Carter CS (2003). Overactive action monitoring in obsessive-compulsive disorder: 
evidence from functional magnetic resonance imaging. Psychol Sci 14: 347-353.

van den Hout M, Kindt M (2003a). Phenomenological validity of an OCD-memory model and the remember/know distinction. Behav Res Ther 41: 369-378.

van den Hout M, Kindt M (2003b). Repeated checking causes memory distrust. Behav Res Ther 41: 301-316.

van den Hout MA, Engelhard IM, de Boer C, du Bois A, Dek E (2008). Perseverative and compulsive-like staring causes uncertainty about perception. Behav Res Ther 46: 1300-1304. van den Hout MA, Engelhard IM, Smeets M, Dek EC, Turksma K, Saric R (2009). Uncertainty about perception and dissociation after compulsive-like staring: time course of effects. Behav Res Ther 47: 535-539.

Volans PJ (1976). Styles of decision-making and probability appraisal in selected obsessional and phobic patients. $\mathrm{Br} J$ Soc Clin Psychol 15: 305-317.

Wiecki TV, Sofer I, Frank MJ (2013). HDDM: hierarchical bayesian estimation of the drift-diffusion model in python. Front Neuroinformatics 7: 14.

Supplementary Information accompanies the paper on the Neuropsychopharmacology website (http://www.nature.com/npp) 